\title{
“TÚ NOS HAS SOÑADO". NOTAS SOBRE EL SUEÑO EN LOS CANTOS CHAMÁNICOS TZELTALES
}

\author{
"You Have Dreamed Us". A Few Notes \\ on the Dream in Tzeltal Shamanic Songs
}

Pedro Pitarch Ramón ${ }^{1}$

Resumen: La revisión del término tzeltal way — sueño— según aparece en los cantos chamánicos permite reconocer algunos aspectos relativos a la naturaleza del sueño entre los tzeltales de los Altos de Chiapas. Esencialmente, el sueño es pensado como un estado ontológico que se contrapone al estado solar de vigilia, en el cual el primero tiene una función agentiva, mientras el segundo ocupa un papel pasivo. Dicho de otro modo, somos soñados por el sueño.

Palabras clave: tzeltales, mayas, sueños, cantos chamánicos.

Abstract: A revision of the Maya-Tzeltal term way (dream) as it appears in shamanic songs allows reconstructing some details relative to the nature of dream among the Tzeltal of Highland Chiapas. Essentially, dream is thought of as an ontological state which contrasts with the solar waking state, in which the first has an agentive function, while the second plays a passive role. Otherwise said, we are dreamed by the dream.

Keywords: Maya-Tzeltal, dreams, shamanic songs.

\footnotetext{
${ }^{1}$ Universidad Complutense de Madrid. Correo electrónico: petul@telefonica.net Fecha de recepción: 3107 17; Fecha de aceptación: 200917.

(cc) BY-NC-ND Páginas 21-42.
} 
Estas notas se refieren al sueño tal y como éste aparece en los cantos chamánicos en tzeltal, lengua maya hablada en las tierras altas de Chiapas, en el sureste de México. Qué es el sueño, esto es, cuál es su naturaleza; no en qué se sueña, o cómo es la narrativa del sueño o cómo se interpretan los sueños, sino cuál es el concepto indígena del sueño. El argumento, en breve, es que el sueño no es tanto una acción cuanto un estado del ser, una condición ontológica; una condición en que la persona no sueńa, sino que es ganada por el sueño, no duerme sino que se es dormida. De hecho, dormir y soñar —a diferencia de lo que sucede en las lenguas europeas - resultan estados equivalentes, comúnmente designados por un mismo verbo: way.

Si el verbo way significa dormir y soñar, su campo de significados se extiende sin embargo a aspectos aparentemente muy distantes de estas acciones primarias. Significa noche u oscuridad tanto en un sentido literal como metafórico. El tiempo prenatal es el tiempo del sueño, como también pertenece al sueño la época mitológica pre-solar; en realidad, un no-tiempo. En un sentido algo distinto, way es sinónimo de inteligencia — como tener la capacidad de intelección-, de visión - como capacidad de ver en la distancia y a través de las cosas, y por tanto de conocer, tal y como las divinidades "ven"- y de posesión de nahuales —esto es, el alma animal que posee ciertos poderes y se desenvuelve en el dominio del sueño.

Lo que subyace a todos estos significados es el hecho de que, como notaba más arriba, el sueño no es tanto una actividad cuanto un estado ontológico (Pitarch, 2013a). Este estado se denomina en tzeltal el Otro lado - chalamal - En contraste con el mundo que habitamos en la vigilia — jamalal, el dominio del sol y de la luz-; el Otro lado representa el dominio de los espíritus, las divinidades, los muertos — la muerte-, las almas. Un estado virtual, intensivo, heterogéneo, infinito. Un modo de existencia sumamente inestable que ignora las coordenadas cronométricas y carece de tiempo y espacio. El alma que "sale" del cuerpo durante el sueño simplemente cobra autonomía para desenvolverse en un estado ilocalizable. A juzgar por los cantos chamánicos, el modo de orientarse en el Otro lado — su balizamiento, por así decir- depende más bien de datos sensibles tales como los movimientos, los colores, los 
sonidos, la temperatura, la luminosidad. En ese estado las divisiones no son nítidas o transparentes, pues se encuentran en una situación de indeterminación, esto es, en un estado que no es ni de mezcla ni de separación. Oposiciones elementales del lado solar tales como presente y pasado, cercano y lejano, vida y muerte, calor y frío, animal y humano, masculino y femenino, hermano mayor y hermano menor, indígena y europeo, etc., alternan incesantemente. Es justamente esta inestabilidad de tiempo y espacio la que caracteriza las experiencias oníricas.

El mundo de la vigilia —el dominio actual, extensivo, homogéneo resultado de la emergencia del sol- debe extraerse incesantemente de este mundo basal del sueño. Pero aunque el sol permite la vida - provisionalmente - tal y como la conocemos, el dominio del sueño envuelve esta dimensión solar y se encuentra permanentemente presente como el reverso de la existencia. Es un dominio sin fronteras precisas. Durante el día cede y se contrae como resultado de la dilatación de la luz solar - y permanece en las sombras, esa otra manera de llamar a las almas o, lo que es lo mismo, en la niebla espesa, donde los intervalos se desvanecen-. Durante la noche se expande invadiendo el mundo solar, apenas respetando el fuego de los hogares que están permanentemente encendidos — así sea con unas pavesas cubiertas de cenizaporque la luz lunar carece de la fuerza suficiente para crear la estabilidad necesaria para que la vigilia no sea completamente absorbida por el sueño (Pitarch, 2013a: 20-22).

En definitiva, el sueño se cierne permanentemente sobre la vigilia, como la muerte se cierne sobre la vida. O dicho de otro modo, el sueńo no es más que una muerte provisional. Así pues, no es tanto que se sueñe o se duerma como que el estado del sueño se apodera de la persona: la persona contrae el sueńo, del mismo modo que contrae la enfermedad - siempre procedente del Otro lado — o se embriaga (cf. Orobitg, 1998). El sueño atrapa, se adueña de la persona; la persona cede, se entrega, o bien se resiste a ser abducida por el sueño. Como si se tratara de un paréntesis en el esfuerzo por excavar el mundo de la vigilia, el cuerpo y sus sentidos pierden el dominio de la conciencia durante el sueño y el alma pasa a desenvolverse en su estado original. Pero es evidente que debe regresar a tiempo para poder reanudar la vida. 
Un dato suplementario: los lingüistas han observado la orientación pasiva de ciertos verbos en lengua mayas, entre los que se encuentra dormir/soñar. Gilles Polian, por ejemplo, explica cómo en tzeltal los verbos intransitivos se distinguen en agentivos y no agentivos. "[El] verbo nux 'nadar' es agentivo, en el sentido en que generalmente, se trata al sujeto que realiza la acción de nadar como un agente que actúa con voluntad y control sobre esa acción, mientras que el sujeto de ch'ay 'perderse' es un ser afectado por el evento, típicamente contra su voluntad y sin control sobre él” (Polian, 2013: 321-322). El verbo way — soñar/dormir - pertenece a esta segunda clase no agentiva en la cual las cosas suceden fuera de la voluntad y dominio de la persona: un paciente, no un agente.

La tendencia pasiva del sueño humano contrasta con el hecho de que las divinidades son pensadas como soñando activamente. Más aún, como veremos, son los dioses y los espíritus quienes sueñan a los humanos y no los humanos quienes sueñan a los dioses. Es curioso que en el texto de Vicente Torres Lezama — en este volumen - se observe esta misma característica en los sueños de la región andina del Cuzco, donde cuando una persona sueńa con los santos y las vírgenes, en realidad son éstos quienes están soñando con el humano. Hasta donde puedo decir, éste es un aspecto que no había sido observado anteriormente en la etnografía del sueño amerindio. Sabemos que en la mitología indígena no es raro que la creación del mundo sea resultado del sueño de los dioses. Pero la actualización de esta condición original, es decir, que los sueños humanos sean producto del sueño de los personajes sagrados, es algo que no ha llamado la atención etnográfica. El principal hilo conductor de mi argumento es precisamente la intuición de que, en la relación entre los dos lados de lo existente, el papel activo es ejercido por el Otro lado, el dominio del sueño y de los espíritus, mientras que el mundo solar y los humanos que en él habitan juegan un papel simplemente pasivo: el mundo de vigilia es sońado por el Otro lado.

Los comentarios que siguen se basan en cantos chamánicos recogidos por mí en los valles de Cancuc entre 1990 y 2004. Estos cantos son largos textos que pronuncian los especialistas chamánicos en las sesiones de curación y protección de enfermedades. Un dato 
decisivo para el tema de este ensayo - los sueños - es que los cantos llegan a los chamanes precisamente en los sueños. El futuro especialista recibe por parte de los espíritus un grueso libro donde están registrados todos los cantos, el cual queda depositado en su corazón. Los cantos chamánicos, pues, representan un lenguaje trascendente, aquel que pertenece al Otro lado — por esa razón no son fáciles de entender-, es decir, es el lenguaje de los espíritus. Dicho de otro modo, los cantos son sueños, y los fragmentos que veremos aquí son, por tanto, sueños hablando de sueños.

\section{El estado de sueño}

Comencemos por un ensalmo llamado sk'oplaltesel jilel, "palabras para el alma detenida" (Pitarch, 2013b: 110-111). Es un texto de carácter formulario que se puede añadir como epílogo de los cantos de curación dirigidos a liberar el alma retenida por los espíritus. Una vez que — tras largas negociaciones y ofrendas de rescate- el alma es liberada por los espíritus, ésta queda perdida y en un estado de desorientación. El texto del ensalmo procura entonces dirigir el alma extraviada para que se reincorpore en el corazón — "su modesto seno, su modesto cajón”- del cuerpo del que se ha separado. El texto dice:

k'ax ch'ul ora stukeloni

k'ax ch'ul ajk'abal stukeloni

wik' iltel yala sit

wik'iltel yala chikin

ba'ay yala jo'benale

ba’ay yala kajonili

mame ja'uk

ay xanix stsa'nelaloni

ay xanix xenelaloni

yu' un ate sikil witsetike

yu'un ate sikil ajawetike

la'me choj

la'me ik'

t'una metel ak'u

t'una metel apak'
Que discurra el tiempo

que termine la noche,

que llegue con la mirada abierta

que llegue con el oído atento,

a su modesto seno

a su modesto cajón.

Que no sufra

la diarrea

el vómito,

del frío de los cerros

del frío de las montañas.

Ven jaguar

ven viento,

busca tu ropa

busca tu vestimenta. 
mame xiwat

mame xa xi' mut

mame xa xi' chanbalam

mame xa xi' wakax

mame xa xi' kawayu

ay ba'ay la ataj ta bej

na'a meixtel anaj

na'a meixtel alum

na'a meixtel awinkilel

na'a meixtel awajch'alel

na'a meixtel awe'el

ja'me xa na'ixtel to

la'me, la'me

ay ba'ay la ataj ta bej

ja'me xa na'ix anaj

ja'me xa na'ix alum

ja'me xa na'ix achanul

ja'me xa na'ix ak'u'

ja'me xa na'ix apak'

la'me, la'me

la'me

mame xwayat.
No te asustes

no te asustes de los pájaros

no te asustes de los animales

no te asustes del ganado

no te asustes de los caballos.

Debes encontrar el camino y

reconocer tu casa

reconocer tu pueblo

reconocer tu figura

reconocer tu cuerpo

reconocer tu alimento

reconocer ya todo esto.

¡Ven, ven!

Debes encontrar el camino

para llegar a tu casa

para llegar a tu pueblo

para llegar a tu animal

para llegar a tu ropa

para llegar a tu hábito.

¡Ven, ven!

ven

no te quedes en el sueño.

El ensalmo va guiando y alentando el alma para que llegue hasta su pueblo, su casa, su ropa, a la vez que trata de evitar que sufra los peligros del camino y sobre todo que no se asuste, especialmente de los animales —algunos tan exóticos como el ganado vacuno o los caballos-. Pero no debemos suponer que el viaje se produce en el dominio de la vigilia — del mundo solar-, esto es, por una geografía continua. En realidad, se trata de que el alma transite entre el dominio del Otro lado, el estado del sueńo, y el mundo solar en el que se encuentra el cuerpo desanimado. El comienzo del texto no puede ser más explícito: "Que discurra el tiempo, que termine la noche", es decir, que acabe el mundo nocturno sin tiempo, habitado por los espíritus que han secuestrado el alma, y comience a transcurrir el tiempo cronométrico propio del dominio solar.

Sin embargo, para nuestros propósitos aquí, la expresión más significativa es la línea con la que finaliza el texto: "no te quedes en el sueño". La expresión mame xwayat cabría ser traducida por "no 
te duermas", pero si bien se mira, esto carece de sentido tanto por la situación en la que se encuentra el alma como por lo que hemos observado más arriba acerca del carácter del sueño. En realidad, el rezador está advirtiendo al alma de que no puede, no debe permanecer en el sueño, esto es, en el dominio del Otro lado. Debe abandonar el mundo del que originalmente surgió durante el nacimiento de la persona y al que regresa periódicamente durante el sueńo, la embriaguez alcohólica, la enfermedad o los momentos emocionales intensos. Es el mundo de la muerte, en el cual la memoria personal — singular- se desvanece progresivamente. De ahí que el rezador insista en que el alma reconozca su lugar en el mundo solar, esto es, se reconozca a sí misma como parte del conjunto de la persona y su entorno solar cotidiano.

El segundo texto es un fragmento de un largo canto titulado chukel ta kurus, "cárcel en la cruz". Como siempre, un alma ha sido secuestrada y encarcelada por los espíritus en el Otro lado, o más precisamente en el umbral hacia el Otro lado que representan las cruces. El rezador se presenta ante una divinidad ofreciendo sus palabras de reconocimiento junto con ciertas ofrendas a cambio de "la mano y el pie" del alma ch'ulel retenida, esto es, su figura completa que consta de veinte dígitos. La mirada de la divinidad — su "parpadeo", como se dice en el canto-, a diferencia de la de los humanos, es capaz de alcanzar todos los dominios del cosmos. En contraste con el caso anterior, este alma ch'ulel debe ser liberada directamente por alguien: una vez identificado el lugar donde se encuentra la cruz en la que está presa, bien la divinidad puede rescatar el alma, bien lo pueda hacer el alma del rezador mismo. En cualquier caso, el fragmento repite la fórmula acerca de que no quede en el estado del sueño, lo que equivale a decir retenido en el otro lado.

jtatati

kajwalati

ja'me bik'itel jti'

ja’me bik'itel ko'tan

tal kabeyix sjelol yok

tal kabeyix sjelol sk'ab padre mío

mi señor,

ésta es mi humilde palabra

éste es mi humilde corazón,

la he venido a ofrecer a cambio de su pie

la he venido a ofrecer a cambio de su mano, 
jtatati

kajwalati

sujtuk me akuxul sit

sujtuk me akuxul elaw

jtatati

kajwalati

ayuk la bit'il xa wilbon

ta yutil ch'ulchan

ta yutil balumilal

xwik'wonoktel sit

yala me'baal xch'ulel

yala me'baal anjel

manchuk teyuk wayal aj

manchuk teyuk chukul aj

ta olil bej abi

ta olil kamino abi padre mío

mi señor,

devuélveme tu viva mirada

devuélveme tu vivo rostro,

padre mío

mi señor,

te ruego que me lo busques

en el interior del cielo

en el interior de la tierra,

que el parpadeo de tu mirada alcance

su pobre alma

su pobre ángel,

que no quede allí en el sueño

que no quede allí retenido

en mitad del camino

en mitad de la travesía

Un tercer y último ejemplo del sueño como estado ontológico procede del fragmento de un canto dirigido a los "madres-padres", métik tatik. Estos espíritus tienen aspecto de ancianos y ancianas con rasgos europeos, de cabellos encanecidos y largas barbas blancas, los cuales, invisibles, se sientan sobre sillas y en torno a mesas en mitad de los senderos del pueblo para beber café. Los humanos que caminan desprevenidos por los caminos pueden derramar inadvertidamente el café de sus pequeñas mesas, dado que estos espíritus resultan invisibles al ojo humano. Como castigo por ello, los espíritus madres-padres cautivan el alma ch'ulel del caminante y no la devuelven hasta que reciben por ella numerosas ofrendas de restitución. - Estos personajes funcionan, de hecho, como espíritus de la tierra que castigan a los indígenas por causa del cultivo del café, obstaculizando las sendas que comunican entre sí a los grupos domésticos.

En lo que sigue el rezador comienza por presentar dichos espíritus: son los que comen, los que tienen — las mesas y el café-, las ancianas, los ancianos. Lo que dicen estos ancianos a continuación tiene un carácter un tanto oscuro: "vamos a comer su ave, vamos a comer su sangre”. Quizá se refiera a una de las almas indígenas que es un ave y a la 
sangre del cuerpo: es conocida la avidez de los espíritus mesoamericanos por la carne y sobre todo por la sangre humana. En seguida el rezador presenta sus ofrendas a través de Jesucristo: objetos amarillos y dorados - posiblemente la luz de las velas en el altar doméstico- junto con el aguardiente. Y ahora menciona lo que ha podido suceder: los espíritus ancianas-ancianos hicieron caer a la persona — esto es, dejaron el cuerpo en posición horizontal - y sustrajeron su alma. El acto de hacer perder la posición vertical - estar erguido sobre las dos piernas, posición característica de vigilia - implica trasladar la conciencia de la persona del cuerpo al alma, y por tanto sumir dicha conciencia en el estado del sueño. Así pues, si bien el sueño apresa a la persona de forma genérica, en este caso son los espíritus quienes trasladan activamente la conciencia de la víctima del estado de vigilia al del sueño.

jayeb we'eloni yo’tikoni jayeb kich'ojtik yo'tikoni jayeb ala me'el yo'tikoni jayeb ala mamal yo'tikoni ya xwe'otik ta yala mut yo'tikoni ya xwe'otik ta yala ch'ich'el yo'tikon xiwan yo'tik balumilal jex xiwan yo'tik balumilal kontrae yos jesukristo chaneb k'anetel yos jesukristo chaneb juchetel ja'niman sjeltay stukeloni jun ach'ul limeta yo’tikoni jun ach'ul baso yo'tikoni jun stukel yilel yo'tikoni sjoyok k'axel yok yo'tikoni bit'il wan ak'ax yak'bonik stsujkulin bit'il wan ak'ax swayabinik ta sti'wan yalib xmexaik yo'tik me'eletik niwan yo'tikoni mamaletik niwan yo'tikoni cuántos son los que comen ahora cuántos son los que tienen ahora, cuántas son las humildes ancianas ahora cuántos son los humildes ancianos ahora. "Vamos a comer su ave" ahora "vamos a comer su sangre" ahora, así dirían los grandes envidiosos así dirían los grandes enemigos.

Dios Jesucristo cuatro amarillos, dios Jesucristo cuatro dorados, para intercambiar solamente junto con una sagrada botella ahora junto con un sagrado vaso ahora, para que los puedan ver ahora. Pasó caminando ahora cuando quizá me lo derribaron cuando quizá le sumieron en el sueño acaso junto a sus mesas ahora de las ancianas quizá ahora de los ancianos quizá ahora. 


\section{Ausentarse del sueño, despertar a la vida}

En los cantos chamánicos el sueño se encuentra asociado a la noche. De hecho el estado de sueńo se utiliza frecuentemente como equivalente de noche, pero con algunos matices. Cuando se emplea el término aj'kabal — noche_- éste designa fundamentalmente un periodo de tiempo, es decir, la fase entre el anochecer y las primeras luces; la fase en que la luminaria está ausente. Sin embargo, cuando se emplea way - sueño- la noche no es exactamente un periodo temporal, sino un estado en el cual la ausencia de sol remite al estado original del cosmos. Como sucede tantas veces en la tradición mesoamericana, la dimensión temporal se subordina a la extensión espacial (cf. Gorza, 2006). Es el estado pre-solar de la mitología, e igualmente el estado prenatal, el no-tiempo anterior al nacimiento, esto es, al alumbramiento; aquí también la ontogénesis recapitula la filogénesis.

En los difrasismos de los cantos es común el empleo del pareado wayxan/kuxan — sueño/vida-, donde el primer término indica que se abandona el sueño y el segundo que se despierta a la vida. Dicho de otro modo, el pareado refiere el nacimiento de la criatura, donde éste consiste en separarse del estado del sueño para pasar al estado de la vigilia. Como ejemplo tomemos el canto llamado smajtan poko méil tatil, "ofrenda para los pasados madres-padres". Se refiere a los ancianos que acaban de pasar su periodo de cargo en el cerro ch'iibal. Se trata éste de un cerro donde habitan las almas ch'ulel de un mismo linaje, el cual se encuentra internamente dividido en trece pisos superpuestos que adoptan una forma piramidal, los cuales se dividen a su vez en numerosos compartimentos. En el piso superior de la montaña, el décimo tercero, viven las almas de los seres humanos de más de cuarenta años, los "madres-padres"; en el duodécimo habitan las almas de entre veinte y cuarenta ańos de edad; y en el undécimo viven los "grandespequeños", los que tienen menos de veinte años. En el preciso momento del nacimiento de un niño en el mundo solar, un doble de su alma aparece en el cerro.

En el siguiente fragmento del canto el rezador está intercediendo por el alma de una criatura recién nacida, para lo cual debe ofrecer ciertas sustancias - aguardiente, velas, incienso, flores, a veces tortillas- a 
toda la jerarquía de almas del cerro. Este canto particular está dirigido a los ancianos que acaban de pasar su cargo en la montańa, puesto que debe cerciorarse que todas las almas queden satisfechas con sus regalos y contribuyan así al bienestar del alma del nińo. Aquí comienza por informar a las madres-padres que el bebé ha salido del cuerpo carnal de su madre - en el mundo solar-; entonces nombra a las madrespadres como "compradores/recibidores" del alma del bebé con el fin de que la abracen/arropen. Lo que nos interesa, sin embargo, es lo que cita a continuación : "así ha despertado del sueño, así ha despertado a la vida”. Ha abandonado el estado del sueño y ha pasado al estado solar de vigilia. Ha nacido. Lo que sigue a este pareado es también significativo: "ya cruzó al sagrado pueblo, ya cruzó al sagrado lugar". Nacer equivale a cruzar el umbral desde el Otro lado hasta el pueblo, el lugar en el que vive ahora. - Aquí, pueblo indica no algo opuesto al espacio deshabitado, sino un lugar sobre la superficie de la tierra.- Más aún, el verbo que emplea para cruzar es jet, cuyo significado más preciso es "bifurcar algo", lo cual probablemente se refiere a que la criatura, al nacer, se bifurca su alma en la que se encuentra en el corazón del cuerpo y en la que se halla en la montańa ch'iibal. ¿Cómo ha aparecido el bebé? ¿Cómo ha despertado del sueño? Porque "salió de ella, salió de su parte", del cuerpo de su madre.

sbak'etal sme'

sbak'etal stukel

ja wala manojel

ja wala ich'ojel

lekuk la petbil

lekuk la loch'bil

jichuk la awayxan

jichuk la akuxan

bit'il la sjetumtay

ch'ul lum

bit'il la sjetumtay

ch'ul k'inal

bit'il stukel el cuerpo de su madre

de su carne solamente, así es humilde Comprador así es humilde Recibidor, que se encuentre bien abrazado que se encuentre bien arropado, así ha despertado del sueño así ha despertado a la vida, cómo ya cruzó al sagrado pueblo, cómo ya cruzó al sagrado lugar, cómo ha sido, 
lok' ta swenta

lok' ta stojol salió de ella

salió de su parte

Un segundo difrasismo comúnmente empleado en los cantos es wayl patajsakub, esto es, sueño/amanecer, el cual designa la transición entre el estado del sueño y el de vigilia, pero significa también, como veremos, la aparición de cosas que se encuentran en estado latente y se vuelven actuales.

El siguiente fragmento del canto pasko, "Pascua", es dicho por el mayordomo principal del santo homónimo, una imagen aparentemente de Jesús de Nazareth que los tzeltales asocian con la Navidad y el solsticio de invierno. Comienza por mencionar a los cuatro mayordomos participantes — “ya estamos todos, los que hemos sido llamados, los que hemos sido invitados"- - Han sido llamados por los espíritus para "hacer florecer, para perfumar" el periodo de celebración del santo en cuestión. Pero sobre todo han sido llamados para "hacer visible", esto es, "apartar del sueño y revelar al amanecer" la caja donde se guarda la imagen de Jesús y el altar con arcos donde se depositan sus objetos rituales. Este último - empleado a lo largo de Mesoamérica- es una plataforma elevada provisional rematada por dos arcos y que representa la arquitectura del mundo solar (cf. Ochiai, 2004). Dicho de otro modo, la apertura de la caja para sacar la imagen de Jesús y la construcción de la plataforma elevada con arcos es tratada como una transición entre el sueño y las primeras luces. La utilización de objetos rituales es una actualización del estado virtual. Hacer aparecer algo no es crearlo, es decir, producirlo de la nada, sino traerlo del sueño, revelarlo a la luz: consiste en actualizar la existencia virtual del sueño.

bats'il bankil

binti ya kutik

mach'a ya jmaliytik

mach'a ya jna'liytik

jo'otik nax ya'ayel

yik'awotik

spayawotik

ta xnichimtesel auténtico hermano mayor,

qué vamos hacer

a quiénes vamos a esperar

a quiénes vamos a conocer,

ya estamos todos

los que hemos sido llamados

los que hemos sido invitados,

para hacer florecer 
sbujts'antesel

ch'ul ora stukel

ch'ul k'aal stukel

bit'il chiknaj

waynax stukel

xpatajsakub stukel

te patalte'

te k'ololte'

yu'un pasko

yu'un jesus

jme'tatate para perfumar

el sagrado tiempo solamente

el sagrado día solamente.

Cómo hacer visible,

extraer del sueño

revelar el amanecer,

la caja

el arco,

de Pascua

de Jesús,

mi Madre-Padre

\section{La inteligencia del sueño: nahuales}

La relación de equivalencia entre el sueño, la inteligencia y los nahuales es afirmada explícitamente en los cantos chamánicos. "Tener el sueño" guarda relación con la idea de inteligencia, esto es, como capacidad de visión y conocimiento. Quienes tienen el sueño poseen un tipo de inteligencia, de comprensión, relacionada con el hecho de que conocen o se desenvuelven en el estado Otro. Existe una inteligencia asociada al cerebro, que es aquella que rige principalmente durante la vigilia, pero existe también una inteligencia del corazón — o penetración del corazón - que es precisamente aquella relacionada con el sueño.

Tener la capacidad del sueño está a su vez estrechamente asociado con la posesión de nahuales, es decir, almas animales. Los seres humanos poseen una capacidad de visión muy limitada, a diferencia de los espíritus para los cuales nada es opaco y su vista alcanza distancias larguísimas. Pero en la medida en que ellos mismos son espíritus y se encuentran del lado del dominio del sueño, los nahuales conceden a la persona de la que forman parte esa visión ilimitada. Desde este punto de vista, el sueño se separa del cuerpo humano y adopta la perspectiva, por ejemplo, de un animal. Kevin Groark (2009) ha notado cómo en la narración tzotzil de los sueños, el objeto del sueño tiende a ser pensado como un "not-self", para lo cual la narrativa onírica emplea un amplio abanico de formas gramaticales y expresiones que tienden a separar 
al hablante del sujeto que ha experimentado el sueño, ya sea el alma ch'ulel o un nahual.

Por lo demás, historiadores y epigrafistas de la civilización maya precolombina (Houston y Stuart, 1989; Velásquez, 2013) han advertido la asociación del glifo que se lee como WAY, esto es, sueño, con la presentación de nahuales o "coesencias" animales, generalmente, pero no únicamente, asociadas con felinos. También el vocabulario tzeltal del siglo XVI del fraile dominico Domingo de Ara (1986: 402-403) subraya — sin duda, por razones de su profesión — esta asociación del sueño con el dominio de las capacidades invisibles: uayaghelal, "brujería”; uayaghom, "brujo" — literalmente, "el que sueña”-; uayaghelal pazoghel, "nigromancia"; uayich, "visión de sueños".

El fragmento que sigue pertenece a un canto llamado "vida para los nahuales de río” —xkuxlej chanul ja-. Estos seres tienen cuerpo de serpiente acuática, pero cuya cabeza es un instrumento metálico: un machete, un hacha, una pala, unas tijeras, y así. En ocasiones estas serpientes se presentan trenzadas formando un petate. Esta especie de nahuales se emplea en erosionar el cauce de los río hasta que produce los deslaves; viven en pozas profundas y lagunas. El rezador pide a las divinidades que concedan una vida larga tanto a este nahual de río como a la persona en su conjunto, puesto que uno depende del otro para vivir. Esto es porque "acaso así fue predestinado, a la sagrada tierra, al sagrado mundo", es decir, el destino de la persona es haber nacido con este nahual. "Así le inventaste quizá, en el vientre, en el seno de su madre, en el seno de su padre", esto es, nació ya con el nahual. El sentido último de la afirmación es que la persona en su conjunto no es responsable de tener nahuales, es una providencia no buscada. Por ello añade que el nahual: "no se compró él a sí mismo, no se introdujo por sí mismo" en el vientre materno, sino que fue la propia divinidad quien decidió su destino y por tanto, en cierto modo, tiene la responsabilidad de mantener la vida del nahual y de la persona. Poseer nahuales proporciona una inteligencia del corazón añadida, pero implica también riesgos, dado que el nahual puede ser dańado o muerto, y el rezador trata de hacer descansar la responsabilidad sobre la divinidad. 
Lo interesante aquí es precisamente el modo en que se refiere al nahual otorgado por la divinidad: "tú le has entregado su sueño, su inteligencia”. Así pues, el nahual representa su sueño y es también la inteligencia del ser humano; por causa de tener un nahual la persona puede soñar, tiene visión, esto es, posee una inteligencia incrementada producto de su capacidad de ver cosas de otro modo inaccesibles.

ja’me ya awil

bik'itel jti'

ja’me ya awil

bik'itel k'otanoni

ja’me ya awil

ch'a'ontel yo'tikoni

takinontel yo'tikoni

sk'anel ate snajtil oraile

sk'anel ate snajtil k'aale

sk'anel ate snajtil jkuxleje

sk'anel ate snajtil jtelamale

skajniwan yu'un jich la spas

smantal ch'ul lumi

smantal ch'ul k'inali

skajniwan yu'un jich la awak'

ta yutil ch'ulchan

la awak'

ta yutil balumilal

chiknaj niwan

ta sjo'benali

ta skajonil me'i

ta skajonil jtati

manix ja’ukla sman sbaj aj

manix ja'uk la xch'ik sbaj aj

ja'nix awak'obey

swayeli

sp'ijili tú lo puedes ver

vengo con mi palabra modesta,

tú lo puedes ver

vengo con mi corazón humilde,

tú lo puedes ver,

vengo amargo ahora

vengo seco ahora,

para pedir para él un tiempo largo

para pedir para él una vida larga

para pedir para él una existencia larga

para pedir para él una ventura larga

porque acaso así fue predestinado

a la sagrada tierra

al sagrado mundo,

porque acaso así le enviaste

adentro del cielo,

le enviaste

adentro de la tierra,

así le inventaste quizá

en el vientre

en el seno de su madre

en el seno de su padre,

no se compró a sí mismo

no se introdujo por sí mismo

tú le has entregado

su sueño

su inteligencia 
La correspondencia entre sueño y nahuales está igualmente expresada en las siguientes líneas de jamal kuxlejal, "vida en el mundo solar", dirigido a proteger a los seres humanos que viven en este lado solar de la existencia. En este tipo de canto se solicita a las divinidades que un paciente que acaba de ser curado chamánicamente permanezca de ahí en adelante protegido de nuevas amenazas de enfermedad. El rezador ruega que el paciente quede en un cerco, en un corral, protegido en su casa. Pero más adelante menciona a los agresores que han causado la enfermedad o que pueden causarla nuevamente: los enemigos, los que tienen nahuales, o lo que es lo mismo, aquellos que han recibido el sueño de las divinidades: "cuántos son los que tienen nahuales, a cuántos les has dado el sueño”. Nuevamente hace responsable a alguna divinidad de haber concedido nahuales a los humanos y por tanto de la necesidad de colaborar en la contención de esas personas - lo cual es, dicho sea de paso, poco probable.

tey ayuk ta smakte'

tey ayuk ta skoral

ak'olokix yo'tan

bujts'anukix yo'tan

jayeb jexi

jayeb kontrai

jayeb mach'a ay slab

jayeb mach'a ay xwayich awu'uni

ja walab aj

ja nich'nab aj

jtatati

kajwalati

jich nime yaxinal ch'ul te'

jich nime yaxinal ch'ul ak'

wayuk lame ch'ul k'inal

wayuk lame ch'ul ora

siketuk swinkilel

siketuk yajch'alel que quede en su cerco, que quede en su corral, que su corazón esté alegre que su corazón florezca. Cuántos son los agresores, cuántos son los enemigos, cuántos son los que tienen nahuales, a cuántos les has dado el sueño, tus hijos tus vástagos. Padre mío, mi señor.

A la sombra del sagrado árbol, a la sombra del sagrado bejuco, que esté en orden su sagrado lugar, que esté en orden su sagrado destino, su cuerpo en calma, su persona en sosiego, 
Pedro Pitarch Ramón

ja’uk la stejk'anbon sbaj

ja'uk la sjayanbon sbaj

tey ayuk ta smakte'

tey ayuk ta skoral

lamal stukel

senel stukel
“Tú nos has soñado”. Notas sobre el sueño en los cantos chamánicos tzeltales

El fragmento acaba con una llamada a que el paciente convaleciente permanezca en calma y en silencio en su cerco. Esto guarda relación con la asociación del Otro lado con el ruido, pues en efecto los espíritus viven una existencia bulliciosa, con ruidos, música y baile incesantes. Sin embargo, todo este ruido y diversión tiene, para los humanos, un carácter sombrío y funesto, pues la algarabía de los espíritus y los muertos engendra la enfermedad en el mundo solar. Es común que en los sueños se escuche algún tipo de ruido intenso, lo que anuncia la presencia de la enfermedad; se escucha, por ejemplo, cortejos de espíritus músicos tocando música cristiana, o estruendos de automóviles conducidos por sacerdotes, camiones guiados por obispos, trenes manejados por jesuitas... En definitiva, el contraste sueño/vigilia implica que si el dominio del sueño se caracteriza por ser extremadamente ruidoso, el mundo de la vigilia debe ser idealmente un dominio silencioso, por más que un silencio absoluto resulte inalcanzable (Pitarch, 2013a: 129-132).

\section{Somos soñados por los espíritus}

El último aspecto de esta revisión del sueño concierne al principio, ya mencionado, de que no somos los humanos quienes soñamos los espíritus y las divinidades, sino que, por el contrario, son los dioses quienes sueñan a los humanos. Es una idea que concuerda bien con el principio según el cual en la relación entre el lado del sueño y el de la vigilia, el primero tiene un papel agentivo y el segundo ocupa una posición paciente.

Es difícil decidir si en los cantos esta idea significa que somos soñados por los espíritus o, de modo más general, somos el sueño de los espíritus. En este último caso, el sueño tzeltal resultaría análogo a lo que los aborígenes del centro de Australia conocen como "The Dreaming" o "Dreamtime". Según la versión clásica de Spencer y 
Gillen (1968) — más tarde discutida y matizada (p. ej. Wolfe, 1991)— el tiempo de los humanos no sería sino un sueño de los ancestros: en el Tiempo del Sueño todos los ancestros de un individuo existen simultáneamente, es el no-tiempo en que se vive antes de nacer y al que se regresa tras la muerte. Las analogías con el Otro lado tzeltal son obvias en el sentido de que el Tiempo del Sueño es el tiempo de la mitología. No soy capaz de precisar lo suficiente como para afirmar que, en una perspectiva tzeltal, el mundo solar que habitamos los humanos es un sueño de los espíritus y los dioses. En todo caso, si el Otro lado ocupa la posición de sujeto en el sueño, los humanos somos el objeto de ese sueño, por tanto, aquello que es sońado.

El fragmento siguiente es incierto al respecto. Con todo, muestra claramente el hecho de que los dioses nos sueñan. Pertenece a un canto, mukul kuxlejal, "gran vida o larga vida", el cual está dirigido a pedir una vida prolongada para los miembros del grupo doméstico. En el fragmento que sigue el rezador se dirige a una divinidad femenina sin especificar, a la cual ruega por una vida larga para el rezador y su familia. En un momento dado le pregunta "¿cuánto tiempo tengo de vida?, ¿cuánto tiempo tengo de existencia?”. Y la razón por la que la diosa conoce la respuesta es "porque tú nos has visto, tú nos has soñado". La expresión no es del todo clara; quizá tiene que ver con la idea indígena - común a la región de Los Altos de Chiapas - de que cuando nace una persona en el cielo se enciende una vela —orail, del español hora, esto es, tiempo de vida o destino- que se va consumiendo a lo largo de la vida hasta que se extingue. No obstante la vela puede ser apagada antes de que la cera se consuma por completo. En cualquier caso, la diosa conoce de antemano el destino de los humanos: ha "visto" ese destino. Pero lo más importante es que lo ha visto porque lo ha "soñado", lo que es decir, la persona existía virtualmente en el sueño de la divinidad antes siquiera de haber sido concebida.

ch'ul senyora me'

ch'ul senyora balumilal

petaotik

loch'aotik sagrada señora madre

sagrada señora mundo,

abrázanos

arrópanos, 
ta xk'axel ch'ul ora

ta xk'axel ch'ul k'aal

ta xk'axel ch'ul ajk'abal

sok jkal

sok jnich'an

sok joy

sok jmajtan

kuxul tat

kuxul kajwal

ch'ul senyora me'chunin

ch'ul senyora alajel

balumilal ku'un

petaotik

loch'aotik

ta jkuxineltik

ta jtelamaltik

maxa wak'otik

ta sk'ab pukuj

maxa wak'otik

ta sk'ab satanas

sok te joye

sok te jmajtane

sok te kale

sok te jnich'ane

jichnix jkal

jichnix jnich'an

jichnix joy

jichnix jmajtan

lekuk ya xk'ax k'aal ku'un

lekuk ya xk'ax ora ku'un

k'alal tinilon

k'alal wuts'ulon

jayeb ora kuxulon

jayeb ora telamon para pasar la sagrada vida

para pasar el sagrado día

para pasar la sagrada noche,

con mis hijos

con mis vástagos

con mi compañera

con mi regalo,

padre viviente

señor viviente,

sagrada señora madrina

sagrada señora de los hijos

de mi mundo,

abrázanos

protégenos,

por nuestra vida

por nuestra existencia,

no nos abandones

en manos del mal,

no nos abandones

en manos de satanás,

con mi compañera

con mi regalo

con mis hijos

con mis descendientes,

así mi hijo

así mi descendiente

así mi compañera

así mi regalo,

que transcurra bien el ciclo

que transcurra bien la vida,

estando así agachado

estando así doblado,

¿cuánto tiempo tengo de vida?

¿cuánto tiempo tengo de existencia?, 
lajuk la awilotik

lajuk la awayotik

senyora me'

senyora kaxail

senyora ch'ul santisima kurus

senyora ch'ul santísima,

talat yo'tik tú nos has visto

tú nos has sońado,

señora madre

señora seno,

señora sagrada santísima cruz

señora sagrada santísima,

ven ahora

\section{Nota final}

Una revisión de los cantos chamánicos tzeltales contribuye a distinguir al menos tres aspectos del sueño indígena, o, más precisamente, del concepto indígena del sueño. En primer lugar, a diferencia del significado común de sueño en español, que designa tanto la acción de dormir como la cosa imaginada durmiendo, el sueño indígena es más bien un estado de la existencia. Como tal, se opone al estado de vigilia y se apodera por momentos de él, colocando a los humanos y el resto de los seres en el lado Otro. En segundo lugar, ese estado del sueño es el no-tiempo anterior al nacimiento y posterior a la muerte, y también el mundo mitológico anterior a la primera aparición del sol. De ahí que, en los cantos chamánicos, salir del sueño es despertar a la vida, así como el abandono del sueño equivale al amanecer, entrar en el nuevo mundo solar que se renueva diariamente. Todo lo que existe, existió o existirá, se encuentra allí, en ese otro dominio, en estado virtual. En tercer lugar, el sueño son los nahuales personales, los cuales proporcionan una "inteligencia" y capacidades añadidas a los humanos de los que forman parte. El sueño reside, pues, fuera del cuerpo humano, y este carácter extra-corporal aleja aún más el control de la conciencia de vigilia de la experiencia del sueño. Lo que subyace a estos tres aspectos, en fin, es que el mundo del sueño es intensivo, en contraste con un mundo pasivo de la vigilia. El sueño es el plano inmanente de la existencia, es todo. 


\section{Bibliografía citada}

Ara, fra. Domingo de, 1986, Vocabulario de lengua tzeldal según el orden de Copanaguastla, Mario H. Ruz (compilador), UNAM, México.

Gorza, Piero, 2006, Habitar el tiempo en San Andrés Larrainzar. Paisajes indígenas de los Altos de Chiapas, UNAM, México.

Groark, Kevin P., 2009, "Discourses of the Soul. The negotiation of personal agency in a Tzotzil-Maya dream narrative", American Ethnologist, vol. 36, núm. 4, pp. 705-721.

Houston, Stephen y David Stuart, 1989, The Way Glyph: Evidence for Co-essences among the Classic Maya, Recent Reports on Ancient Maya Writing, Center for Maya Research, Washington.

Ochiai, Kazuyasu, 2004, "Bajo la mirada del sol portátil: representación social y material de la cosmología tzotzil”, en Johanna Broda y S. Iwanizesqui (compiladores), Arqueoastronomia y etnoastronomía en Mesoamérica, UNAM, México.

Orobitg Canal, Gemma, 1998, Les Pumé et leurs rêves. Étude d'un groupe indien des Plaines du Venezuela, Editions des archives contemporaines, Amsterdam.

Pitarch, Pedro, 2013a, La cara oculta del pliegue. Antropología indigena, Artes de México, México.

Pitarch, Pedro, 2013b, La palabra fragante. Cantos chamánicos tzeltales, Artes de México, México.

Polian, Gilles, 2013, Gramática del tseltal de Oxchuc, CIESAS, México. Spencer, Walter B. y Francis J. Gillen, 1968 [1899], The Native Tribes of Central Australia, Dover, Nueva York.

Velásquez García, Erik, 2013, "Nuevas ideas en torno a los espíritus wahyis pintados en las vasijas mayas: hechicerías, enfermedades y banquetes oníricos en el arte prehispánico”, en Erik Velásquez García (compilador), Estética del Mal: conceptos y representaciones, UNAM, México.

Wolfe, Patrick, 1991, "On being Waken Up: The Dreamtime in Anthropology and in Australian Settler Culture", Comparative Studies in Society and Culture, núm. 33, pp. 197-224. 